\title{
Added Mass of Beam Vibration On Fluid With Finite Amplitude
}

\author{
Ping Liu ${ }^{1, a}$, Wei Miu ${ }^{1, b}$ \\ ${ }^{1}$ School Of Architecture And Civil Engineering, Jiangsu University of Science and Technology \\ aliupinghaiyan@163.com, b382349957@qq.com
}

Keywords: Euler beam, added-mass ratio, fluid-structure interaction

Abstract: As the strong non-linear characteristic of Naveir-Strokes equation, the theory of fluid-structure interaction (FSI) has a slow progress. This paper solves a two-dimensional FSI problem in which a Euler-beam vibrating in fluid. The beam satisfies Euler-Benelli beam assumption and fluid is a non-vertox, non-viscosity fluid. On the basic of above assumption and with Fourier series method, the governing equation is reduced and the theoretical solution is obtained. From the solution, the frequency of beam vibration is in accordance with the nature frequency of the beam vibrating with a "added mass". And on the axis direction of beam, velocity and pressure is a simple harmonic quantity, on the normal direction of beam, velocity and pressure is in a quantity in the exponent. On the field of time, all the physical quantity are the harmonic function. Besides, the numerical simulation was carried out and the result agreed with theory predictor.

\section{INTRODUCTION}

Beam vibration submerged in fluid have widely application in engineering, from ships in oceans to micro-electromechanical system (MTMS) devices[1,2]. Many researchers have studied on vibration of such beams. Excellent reference source may be found in the open literature[3-6]. Because of the complexity of Nervier-Stoke equation, there is almost no progress in the theory. And usually the numerical method are used to solve beam's vibration interacted with fluid[7]. As well known that numerical method is suitable for a varied application and solution is got fast. But, unfortunately, solution with numerical method is impacted with quality of grid and discrete scheme and so on[8-10]. Thus the entire program nowadays could not have accuracy and stable solution. Besides, numerical solution could not know the principle rule of problem. So, researching in theory aspect is very significant.

Schlick firstly do some study on the vibration of ship submerged in water at 1884 and about 40 years ago, Lewis F.M[11, 12] does a detailed researched on the vibration of ship submerged in quiescent water. Simultaneously, Lewis F.M raises a "added mass" method to consider the impact of fluid. Lewis explain the "added method" in physical aspect and succeed in some filed.

H.Minami[13] firstly solve a vibration of membrane in fluid. It is based the hypothesis that the width is very small so the fluid flow from upper side to lower side with vertex style. The hypothesis is that the added mass distributed uniformed along the membrane. On above hypothesis, H.Minami obtains added mass $0.68 \rho L$. The deducing progress also may apply in beam. Shahrokh Hosseini-Hashemi[14] studied vibration of plate submerged in fluid. It does not assume the simply hypothesis that the wet and dry mode shapes are the same. Daidola, Hohn Chris[15] derived the equation for the Euler-Beam oscillating in stationary fluid. It seems that research papers related to the magnitude of the added mass of vibrating beam and analysis solution is very few.

The objective of this paper is to investigate analytically the fundamental properties of such added mass of a Euler-beam oscillating in unconfined fluid and the properties of fluid. A natural vibration model of the two-dimensional problem, which is a horizontally supported initially plane beam vibrating in a $\mathrm{x}-\mathrm{y}$ plane with finite amplitude, is used as the object for this analysis (Fig-1). In order to get a simple and accuracy solution, Fourier series and variables separation method are used. Finally, the quantity of added mass and the approximate distribution of fluid are investigated in this paper; also the accuracy expression of wet frequency is given.

The result is obtained from the above-mentioned method, based on Fourier series approach, are compared with those obtained from numerical solution and vortex distribution modeling approach. 


\section{The METHOD OF ANALYSIS}

In a static state, a two-dimensional beam is supported horizontally at the end, with distance L between the supports. The mass per unit is denoted by $\rho_{s}$. An x-y coordinate system having the origin at one of the points of support is defined as showed in Fig-1. The beam is assumed oscillating in a sinusoidal type. The $\mathrm{x}$-axis is oriented in the direction of length of the horizontal beam. The expression of the displacement $U$ at any point $\mathrm{x}$ any time is

$$
U=u(x) \sin (w \cdot t), 0 \leq \mathrm{x} \leq \mathrm{L}
$$

Where $\mathrm{u}(\mathrm{x})$ is the oscillating shape of beam, and $\mathrm{w}$ is the circular frequency of oscillating.

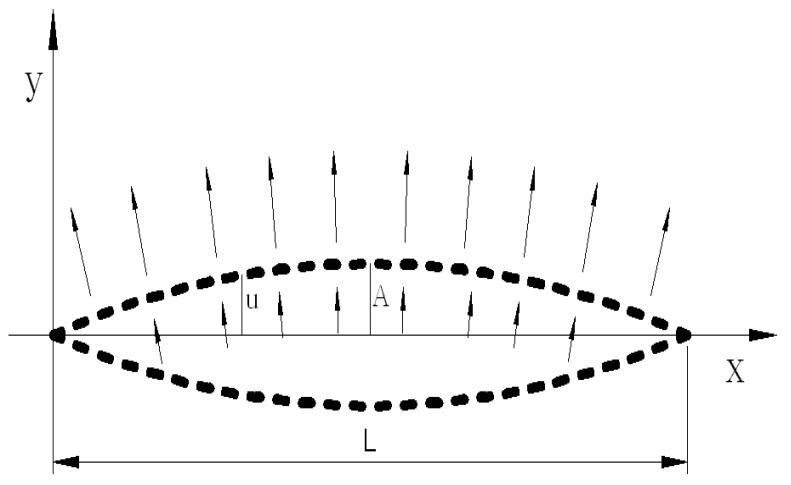

Fig-1 Definition of coordinate and analytical modeling with no vortex hypothesis.

As assumed the fluid is no vortex and no viscid, thus consider both beam and Navier-Stoke equation, it can obtain the governing equation of vibration of beam[16]:

$$
\begin{aligned}
& E I \frac{\partial^{4} U}{\partial x^{4}}+\rho_{s} \frac{\partial^{2} U}{\partial t^{2}}=\rho_{w} \frac{\partial \Phi}{\partial t} \\
& \frac{\partial^{2} \Phi}{\partial x^{2}}+\frac{\partial^{2} \Phi}{\partial y^{2}}=0
\end{aligned}
$$

Where E, I are the elasticity modulus and inertia modulus respectively. $\rho_{s}, \rho_{w}$ the density of beam and water respectively, $\Phi$ the potential function of fluid which satisfied with Laplace Equation. And the corresponding boundary is as follows:

(1) For beam, there have to satisfied $\frac{\partial^{n} U}{\partial^{n} x}=0$, and $n=0,2[15]$, when $x=0, L$.

(2) For flow, $\frac{\partial \Phi}{\partial n}=\frac{\partial \Phi}{\partial y}=v=\frac{\partial U}{\partial t}$, when $\mathrm{y}=\mathrm{U}$, noticed that the $\mathrm{U}$ is not a constant value but the function of time and the direction of $U$ is the $Y$ direction. Besides, when $y \rightarrow+\infty$, there should be $\Phi<+\infty$, that is to say, $\Phi$ is a limited function.

Noticed that, the beam is oscillating with frequency of w, so it reasonable assume that the flow is varied with same frequency. Thus, let the potential function be the type $\Phi=\phi(x, y) \sin (w \cdot t+\varphi)$, and $\varphi$ is the phase angle.

The proceeding to solve the equation is detailed descripted as follows. Let $\phi=f(x) g(y)$, and substitute it into equation (2) and obtained:

$$
f(x)=A_{1} \sin (p \cdot x)+A_{2} \cos (p \cdot x), g(y)=B_{1} e^{p y}+B_{2} e^{-p y}
$$

Where, parameter $p$ is a variable real value.

Substitute the infinite boundary condition, it may fixed that $B_{1}=0$, and to satisfied boundary condition (1), it may fixed that $A_{2}=0$, and

$$
p=\frac{k \pi}{L}, \text { where } \mathrm{k} \text { is arbitrarily positive number, so, } \phi=\sum_{k=1}^{\infty} A_{k} \cos \left(\frac{\pi}{L} k x\right) e^{-\frac{\pi}{L} k y}
$$


Because the flow velocity of beam is the same with it of beam. Substitute condition-(2), therefor,

$$
\sum_{k=1}^{\infty}-\frac{k \pi}{L} A_{k} \cos \left(\frac{\pi}{L} k x\right) e^{\frac{-\pi}{L} k u} \sin (w \cdot t+\varphi)=w \cdot \cos (w \cdot t) u(x)
$$

So, the coefficient of Fourier series may be fixed, which is the shape function's cosine spread coefficient, the detailed expression is

$$
A_{k}=-\frac{L w}{k \pi} \int_{0}^{L} \cos \left(\frac{\pi}{L} k x\right) u(x) d x
$$

And the phase angle is $\pi / 2$, thus, $\Phi=\sum_{k=1}^{\infty} A_{k} \cos \left(\frac{\pi}{L} k x\right) e^{-\frac{\pi}{L} k y} \cos (w \cdot t)$

Substituting the equation (5) to equation (3), it will concluded

$$
\frac{\partial^{4} u(x)}{2^{4}} \sin (w t) \frac{\rho_{s}}{E} w^{2} u(x) \sin (w t)=\frac{-\rho_{w}}{E} w^{2} \sin (w t) \sum_{k=1}^{\infty} A_{k} \cos \left(\frac{\pi}{L} k x\right) e^{\frac{\pi}{L} k t}
$$

The $\sin (w t)$ in the expression above are not always equate zero, thus the item $\sin (w t)$ may divided simultaneously by both side. And so it shows that the left of equation is differential equation of vibration beam and the coefficient of another side is an integration of shape function of beam with coefficient $A_{k}$. With regard of finite amplitude of deflection, let $\exp \left(-\frac{\pi}{L} k u\right)=1$ may not affect large error. The equation above may simplify as follows,

$$
\frac{d^{4} u}{d x^{4}}-\frac{\rho_{s}}{E I} w^{2} u=\sum_{k=1}^{\infty} \int_{0}^{L} \cos \left(\frac{\pi}{L} k x\right) u(x) d x \cdot \cos \left(\frac{\pi}{L} k x\right)=\frac{\rho_{w}}{E I} \frac{L w^{2}}{k \pi} u(x)
$$

\section{SOULTION}

Equation (6) is a typical Liville-Sturm eigenvalue equation. Compared with the vibration equation of beam in vacuum, equation (6) is just like a beam whose quality is increased. The detailed expression is $\rho_{w} L / k \pi$. This is the theory foundation of "added method" for the problem of oscillation beam. by the way, the result also applied in the problem of oscillation membrane. And besides, it can conclude that the deflection shape of vibration is the same with that oscillate in vacuum. And the oscillation frequency is $w_{j}=\left(\frac{\pi}{L} j\right)^{2} / \sqrt{\left(\frac{\rho_{w} L}{k \pi}+\rho_{s}\right) / E I}$ which is lower than that in vacuum.

On the conditions of simple supported boundary, sine series $\sin \left(\frac{k \pi}{L} x\right)$ satisfied the boundary, therefore it may expand initial conditions to sine series. And thus, the solution will be in superposition of different modal of vibration respected with frequency. The detailed expression is $u(x)=\sum_{1}^{\infty} u_{k} \sin \left(\frac{k \pi}{L} x\right) \sin \left(w_{k} t\right)$.

And coefficient $u_{k}$ can be determined by initial condition.

$$
\text { Assume } \mathrm{t}=0 \text {, there are } u(x)=0 \text { and } u^{\prime}=\sin \left(\frac{\pi}{L} x\right) \text {. }
$$

to checking the solution, set a simple initial condition as above and the solution is

$$
U=\frac{1}{w_{1}} \sin \left(\frac{\pi}{L} x\right) \sin \left(w_{1} t\right), \Phi=-\frac{L}{\pi} \sin \left(\frac{\pi}{L} x\right) e^{-\frac{\pi}{L} y} \cos \left(w_{1} t\right)
$$

Therefore, velocity and pressure is 


$$
V_{x}=-\cos \left(\frac{\pi}{L} x\right) e^{-\frac{\pi}{L} y} \cos \left(w_{1} t\right), V_{y}=\sin \left(\frac{\pi}{L} x\right) e^{-\frac{\pi}{L} y} \sin \left(w_{1} t\right), p=-w_{1} \rho \frac{L}{\pi} \sin \left(\frac{\pi}{L} x\right) e^{-\frac{\pi}{L} y} \sin \left(w_{1} t\right)
$$

\section{VERIFICATION}

To verifying the solution, numerical solutions are compared with the results. Weak coupling method is used to obtain numerical solutions and as for structure aspect, calculated with FEM program, as for fluid aspect, calculated with CFD program. Thus, Ansys is chosen for FEM program and Fluent is chosen for CFD program. In Ansys, beam3 element which is pined to both end has been used, besides, the length of beam is set to 50, Yang's modulus $2.0 \times 10^{11}$, Poisson's ratio 0 , area of beam section is set to 1 . Theoretically, the first frequency of beam is $w_{1}=\left(\frac{\pi}{L}\right)^{2} / \sqrt{\frac{m}{E I}}=5.77$. Element of fluid is applied by four node element and density of fluid is set to 100, furthermore, the fluid is set to idea fluid. When both side of beam are fluid, the pressure is two times of equation (8). Considering with fluid, so, the first frequency of beam is $w_{1}=\left(\frac{\pi}{L}\right)^{2} / \sqrt{\frac{\rho}{E I} \frac{2 L}{k \pi}+\frac{m}{E I}}=4.86$. And initial boundary is the same the expression-(7), that is to say, when $t=0$, the initial displacement is zero and the initial velocity is a sine wave with amplitude 1 .

The displacement of mid-beam to time is showed as Fig- 2 and when $t=0.4 \mathrm{~s}$, the shape of beam vibrating is showed as Fig-2 right. From Fig-2, it may concluded that the frequency of beam is $2 \pi / 1.58=3.97$ which is lower than that of no interaction. The theoretical solution is 4.86 , compared with numerical solution, error is $18 \%$. As for amplitude, because of the initial condition and the principal of conservation of energy, the amplitude is not the value $1 / w_{1}=0.234$ but the value which times the ratio of mass $m / M w_{1}=w_{1} / w_{0}^{2}=0.129$. In the solution of numerical method, the amplitude is 0.117 , consequently error is $9 \%$. Besides, from the fig- 2 , it can obtain that the shape of beam in the period of oscillating will not be changed.
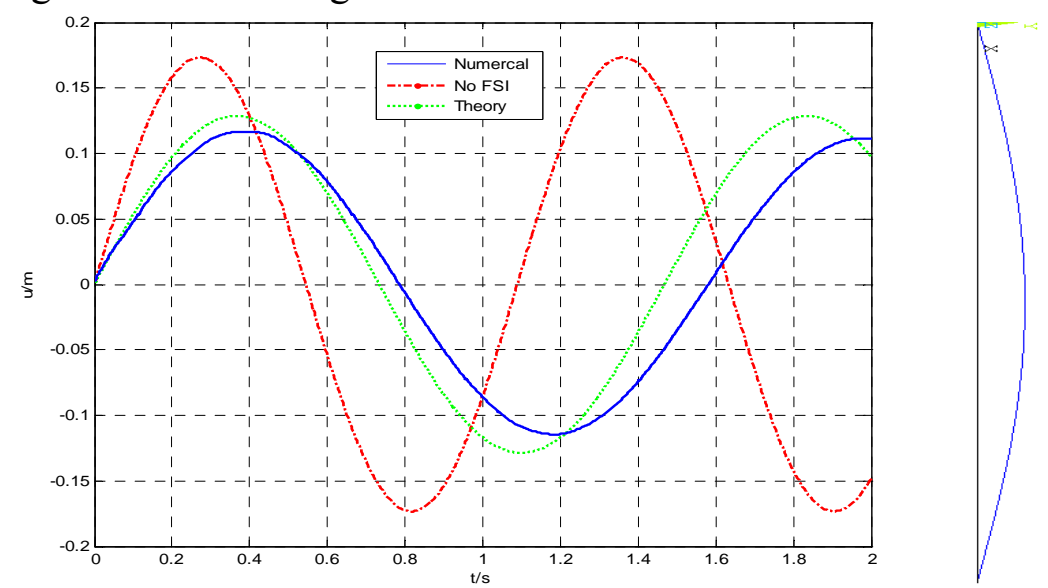

Fig-2 displacement of the mid-node(left), displacement map when $\mathrm{t}=0.4 \mathrm{~s}$ (right)

Fig- 3 shows the contour of $\mathrm{x}$-velocity while $\mathrm{t}=0.4$. it shows that, $\mathrm{x}$-velocity approximately be the cosine shape which theoretically is the shape of function $V_{x}=-\cos \left(\frac{\pi}{L} x\right) e^{-\frac{\pi}{L} y}=$ constant. At the point of beam end, fluid velocity and gradient of velocity is up to the max value. Noted that, because of the hypothesis, the theoretical solution is not satisfied between the field $x \notin(0, L)$. Fig- 3 is the contour of y velocity while time is 0.4 second. Theoretically, y velocity is the shape of function 
$V_{y}=\sin \left(\frac{\pi}{L} x\right) e^{-\frac{\pi}{L} y}=$ constant. Compared with numerical solution, it predicted well. Moreover, in the Fig-3, the closed curve are trace of particle, the closed shape shows that, fluid flows from a side of beam to the other side which shows that the floated fluid is sucked by the other side. That is mean that whole fluid field are no fluid floated or sucked. This is determined by the principle of conservation of mass. From physical aspect, it shows that fluid far away from beam is in peace when beam oscillate.
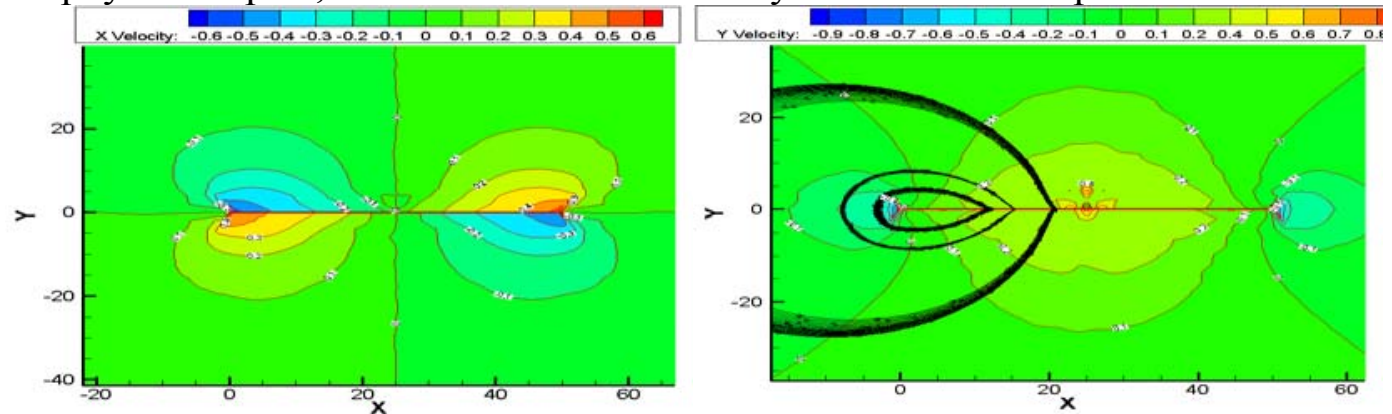

Fig-3 x-velocity of fluid(top) and y-velocity of fluid(low) when $\mathrm{t}=0.4$

Fig-4 is the contour of pressure while time is 0.4 . from equation (7), the maximum pressure is $\rho w_{1} \frac{L}{\pi}=6815$, and in the Fig-4, the maximum value is less than 4000, has a large error compared with theoretical solution. But the trend of pressure is consistent with theoretical predict. From equation (5), it may obtain that both pressure and velocity are sine wave along length of beam and exponential shape along y axis. The pressure trend showed by Fig-4 satisfied these predicting.
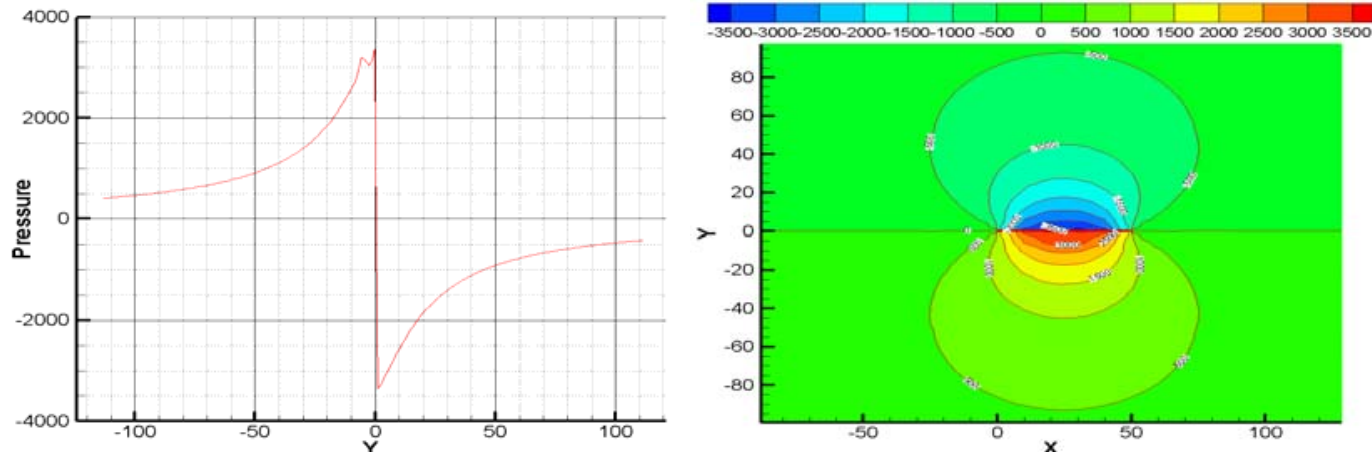

Fig4 pressure of mid-location of beam(top) and pressure of fluid(low) when $t=0.4$

At last of this section, some important should be noted that, almost all of the actual problem are three dimensional. Assuming the beam to be a Euler-beam is to decreased the complexity of problem or it will not find any significant solution in nowadays mathematical level. Furthermore, the theoretical solution equation (5)-(7) is not correct in all the field of definitions. It may see easily from equation (7) that, the solution is a period function to $x$, but at the field $x \notin(0, L)$, the physical fluid evidently are not same with it at the field $x \notin(0, L)$.

\section{CONCLUSIONS}

The added mass of a horizontally supported, initially plane beam undergoing finite amplitude natural vibration in the harmonic fundamental mode is investigated by two-dimensional analysis. Fourier series and variables separation method is applied. From results of numerical analysis, the following conclusions are obtained:

(1) The added-mass ratio uniquely depends on the ratio $\rho_{w} L /\left(k \pi \rho_{s}\right)$;

(2) Velocity and pressure is decrease exponentially along to the y axis which is perpendicular to 
the beam, and to the direction of beam, oscillation are made up by several harmonica wave. At the field of time, oscillation is distributed harmonically. The principal of vibration interacted with fluid is same with that in vacuum. But the frequency is not same which just like the mass of beam increased. The added mass is direct ratio with density of fluid and the length of beam and the wave number.

(3) The pressure on beam is direct ratio with all of the density of fluid, the velocity of beam, frequency of beam. Along the beam, pressure is a harmonica distribution and decreased exponent in the direction perpendicular to beam. in the time field, pressure is made up by harmonica wave.

\section{REFERENCE}

[1]. Maekawa, S. and K. Saito, The effect of ballonet slosh on an airship's longitudinal motion. Transactions of the Japan Society for Aeronautical and Space Sciences, 2004. 47(155): p. 44-50.

[2]. Fukuyama, M., et al., Dynamic buckling experiments of fluid-structure-coupled co-axial thin cylinder. Nuclear Engineering and Design, 1999. 188(1): p. 13-26.

[3]. Tang, D., et al., Multi-Physics MRI-Based Two-Layer Fluid-Structure Interaction Anisotropic Models of Human Right and Left Ventricles with Different Patch Materials: Cardiac Function Assessment and Mechanical Stress Analysis. Computers \& Structures, 2011. 89(11-12): p. 1059-1068.

[4]. Takizawa, K. and T.E. Tezduyar, Multiscale space-time fluid-structure interaction techniques. Computational Mechanics, 2011. 48(3): p. 247-267.

[5]. Kloppel, T., et al., Fluid-structure interaction for non-conforming interfaces based on a dual mortar formulation. Computer Methods in Applied Mechanics and Engineering, 2011. 200(45-46): p. 3111-3126.

[6]. Crosetto, P., et al., Parallel Algorithms for Fluid-Structure Interaction Problems in Haemodynamics. Siam Journal on Scientific Computing, 2011. 33(4): p. 1598-1622.

[7]. Ruck, S. and H. Oertel, Jr., Fluid-structure interaction simulation of an avian flight model. Journal of Experimental Biology, 2010. 213(Pt 24): p. 4180-92.

[8]. Lasiecka, I. and Y.J. Lu, Interface feedback control stabilization of a nonlinear fluid-structure interaction. Nonlinear Analysis-Theory Methods \& Applications, 2012. 75(3): p. 1449-1460.

[9]. Yamada, N., T. Minami, and M.N.A. Mohamad, Fundamental experiment of pumpless Rankine-type cycle for low-temperature heat recovery. Energy, 2011. 36(2): p. 1010-1017.

[10]. Wang, X. and X. Li, Fluid-structure interaction based study on the physiological factors affecting the behaviors of stented and non-stented thoracic aortic aneurysms. Journal of Biomechanics, 2011. 44(12): p. 2177-84.

[11]. Lewis, F.M., The Inertia of the Water Surrounding a Vibrating Ship. Transactions of the Society of Naval Architects and Marine Engineers, 1929.

[12]. lewis, F.M., Vibratioin and Engine Balance in Diesel Ships. 1927: Transactions of the Society of Naval Architects and Marine Engineers.

[13]. H.Minami, added mass of a membrane vibrating at finite amplitude. journal of fluid and structures, 1998. 12: p. 919-932.

[14]. Hosseini-Hashemi, S., M. Karimi, and H. Rokni, Natural frequencies of rectangular Mindlin plates coupled with stationary fluid. Applied Mathematical Modelling, 2012. 36(2): p. 764-778.

[15]. Daidola, J.C., Vertical and Lateral Vibrations of Euler Beams in a Fluid. 1983: university microfilms international.

[16]. Pena, G. and C. Prud'homme, Construction of a high order fluid-structure interaction solver. Journal of Computational and Applied Mathematics, 2010. 234(7): p. 2358-2365.

[17]. Osses, A. and J.P. Puel, Unique Continuation Property near a Corner and Its Fluid-Structure Controllability Consequences. Esaim-Control Optimisation and Calculus of Variations, 2009. 15(2): p. 279-294. 